\title{
Effect of Head Group Size on Permeability of Newton Black Films
}

\author{
RM. Muruganathan ${ }^{* \dagger}$, H.-J. Müller, H. Möhwald, R. Krastev \\ Max-Planck Institute of Colloids and Interfaces, 14424 Potsdam, Germany.

\section{Supporting information:}

Table: A1. The critical micelle concentrations for the surfactants used in this study. All values are taken from Reference ${ }^{\mathrm{i}}$

\begin{tabular}{cc}
\hline Surfactant & $\begin{array}{c}C M C \\
\left(x 10^{-5} \mathrm{~mol} / \mathrm{L}\right)\end{array}$ \\
\hline $\mathrm{C}_{12} \mathrm{E}_{5}$ & 6.6 \\
$\mathrm{C}_{12} \mathrm{E}_{6}$ & 6.8 \\
$\mathrm{C}_{12} \mathrm{E}_{7}$ & 6.9 \\
$\mathrm{C}_{12} \mathrm{E}_{8}$ & 7.0 \\
$\mathrm{C}_{12} \mathrm{E}_{9}$ & 7.1 \\
$\mathrm{C}_{12} \mathrm{E}_{18}$ & 7.4 \\
\hline
\end{tabular}

* Corresponding author: email - Nathan@ chem.fsu.edu

${ }^{\dagger}$ Present address: Department of Chemistry and Biochemistry, The Florida State University, Tallahassee, Florida - 32310, Fax: +1-850-644 8281. 
Table: A2 Area per molecule at the cmc for $\mathrm{C}_{10} \mathrm{E}_{\mathrm{j}}$ and $\mathrm{C}_{12} \mathrm{E}_{\mathrm{j}}$ type surfactants at the air/liquid interface (taken from ref. ${ }^{\text {ii }}$ and ${ }^{\text {iii }}$ ). We also encourage the readers to refer to table 10 and table 11 in reference ${ }^{\mathrm{iv}}$.

\begin{tabular}{cc}
\hline $\begin{array}{c}\text { Surfactant } \\
\text { Area per molecule } \\
\left(\hat{A}^{2} / \text { molecule }\right)\end{array}$ \\
\hline $\mathrm{C}_{10} \mathrm{E}_{2}$ & 40.0 \\
$\mathrm{C}_{10} \mathrm{E}_{4}$ & 43.5 \\
$\mathrm{C}_{10} \mathrm{E}_{5}$ & 46.8 \\
$\mathrm{C}_{10} \mathrm{E}_{8}$ & 67.2 \\
$\mathrm{C}_{12} \mathrm{E}_{4}$ & 42.4 \\
$\mathrm{C}_{12} \mathrm{E}_{5}$ & 45.8 \\
\hline
\end{tabular}

${ }^{i}$ Nonionic Surfactants, Physical Chemistry: Surfactant Science Series, Schick, M.J. (Ed.); Marcel Dekker, New York, 1987.

ii Karraker, K.A., Ph.D. Thesis (1999), Chemical engineering division, UC Berkeley, USA.

iii Persson, C. M., Ph.D. Thesis (2002), Department of chemistry, KTH, Stockholm.

${ }^{\text {iv }}$ Lu, J. R; Thomas, R. K; Penfold, J. Adv. Colloid Interface Sci. 2000, 84, 143. 